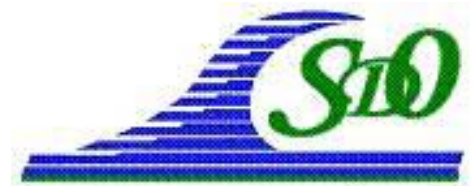

\title{
Une vieille légende : le port d'Ault dans le département de la Somme (Picardie)
}

\author{
Laurent DELABY ${ }^{1}$
}

1. DLIC, Delaby Laurent Ingénieurs Conseils, 143 Boulevard de Magenta, 75010 Paris. l.delaby@yahoo.fr;www.delabylaurent.fr

\section{Résumé :}

S'il est un vieille légende maritime, c'est bien celle d'un port à Ault, dans la Somme en Picardie, un port présent dans les temps anciens que la violence des flots a englouti. Et s'il est bien un vieux projet, c'est bien celui de la (re - ?) construction d'un port à Ault auxquelles depuis des siècles des générations se sont penchées les unes après les autres. L'espérance de voir se réaliser un jour ce port s'est transmise au travers des temps. Nous proposons dans ce papier d'étudier, à notre tour, la faisabilité de la construction de ce vieux rêve et de cette vieille espérance

Mots clés :

Légende - Port de Plaisance - Port de pêche - Modélisation numérique - Histoire Port - Construction écologique - Etude de vagues

\section{Introduction}

Les ports sont d'importants facteurs de développement pour les communes qui les hébergent, ils sont souvent des "portes" ouvertes sur la mer et ses richesses, certes, mais aussi des portes ouvertes sur l'autre, le voyage, le commerce, l'échange, le partage, la rencontre. Ils ouvrent sur la subsistance et la vie au travers la pêche et le commerce, ce sont les aspects "économiques et sociaux". Ils ouvrent sur l'aventure et l'ailleurs au travers les voyageurs qui partent découvrir, apprendre, rencontrer, ce sont les aspects "humains et culturels".

Quel enfant n'a pas rêvé devant des dessins de ports, le déchargement des marchandises venues d'autres latitudes, les histoires de navigateurs, d'explorateurs, de tempêtes, les légendes fantastiques? Il est un village qui au travers des temps parvient à transmettre cet esprit de jeunesse et de rêve, rêvant depuis des siècles de reconstruire son port, un port qui lui permettrait d'aller pécher et d'accueillir les voyageurs au long cours. Il est un village qui doit à la mer son histoire, la mer lui a tout donné, et lui a beaucoup repris aussi, en érodant doucement les falaises sur lesquelles il est construit.

Ce projet de port à Ault dans la Somme (Picardie) n'est pas totalement utopique comme nous allons le voir, et il s'inscrit quelque part dans le mouvement de la nature : si les ports en fond de la Baie de Somme toute proche s'ensablent doucement, n'est il pas naturel de chercher à construire des ports en remplacement plus près de la mer ? 


\section{Le port de Ault : une vieille légende}

Il semble qu'il ait existé un port à Ault dés le $9^{\text {ème }}$ siècle, ainsi un certain Guilmeth dans son opuscule sur Ault, écrivait en 1850: "L'abbaye de Saint-Valery possédait à cette époque le bourg tout entier, son sol, son port, son église et ses habitants".

Idéalement situé devant une zone de pêche féconde entre Ault et Cayeux, le Port de Ault aurait compté au Moyen-Age jusque 80 barques et aurait été l'un des plus importants centre de pêche du nord de la France, alimentant jusque Paris par les "chasses marées" qui arrivaient "rue du Faubourg Poissonnière" dans le prolongement de l'actuelle nationale 1 .

Il semble que ce port ait été situé dans la zone dite du "Hâble d'Ault", à l'ombre du poulier en formation qui arrive aujourd'hui au Hourdel, à quelques kilomètres de là dans la Baie de Somme. Le mot hâble semble évoquer ce passé en venant lui-même "hâvre" et en signifiant port ou zone de refuge. Il semble aussi, en suivant les descriptions successives des auteurs, que le port ait suivi le développement du poulier dont la croissance rapide vers Cayeux déplaçait au fur et à mesure l'abri, et au bout duquel nous trouvons aujourd'hui le port du Hourdel, à l'entrée de la Baie de Somme.

Les hommes ont sans doute essayé de fixer ce port, sans doute y sont ils parvenus par intermittence, et sans doute aussi les tempêtes les plus fortes ont elle réduit leurs efforts à néant suivant des intervalles réguliers, comblant de galet les rades et détruisant les ouvrages, les jetées et les épis.

\section{Présentation de la cote picarde et de Ault}

Le littoral picard est une zone en constante évolution et le bilan sédimentaire y est contrasté. Les côtes en cours d'érosion encadrent la baie de la Somme et celle de l'Authie où la sédimentation prédomine. Le trait de côte a tendance à se déplacer au dépend du domaine continental dans les zones en situation d'érosion, ou du domaine marin dans les zones en sédimentation.

L'érosion des côtes est sensible au Sud, le long des falaises crayeuses: la station d'AultOnival est menacée de disparition sans travaux de lutte contre la mer. Le cordon littoral de galets lui aussi subit les attaques marines; sa destruction partielle entraîne l'inondation des Bas-Champs, comme cela a été le cas en 1990. La protection des BasChamps demande ainsi un entretien régulier du cordon (ANTEA, 1996). Au Nord, c'est l'érosion du haut de plage au niveau des stations balnéaires de Quend et Fort Mahon qui pose problème.

Le colmatage des estuaires, et en particulier celui de la Baie de Somme, a également des conséquences préoccupantes sur la gestion de ces espaces. De vastes surfaces de zones tidale disparaissent chaque années; les schorres s'étendent au dépend des slikkes, les zones où prospèrent les coquillages, zones de nourriceries des oiseaux marins, s'amenuisent. Les chenaux desservant les ports se rehaussent, rendant l'accessibilité de plus en plus aléatoires (BINET, 1994). 


\section{XI ìmes Journées Nationales Génie Côtier-Génie Civil}

Les Sables d'Olonne, 22-25 juin 2010

La plage entre Onival et Cayeux est formée de galets de silex et de sable composé de grains de quartz d'origine marine, de débris de silex et de coquilles. L'estran est une plage de sable qui recouvre une plate-forme rocheuse d'érosion marine faiblement pente vers le large $(0,5$ à $1 \%)$ et montre des formes d'érosion karstique soulignant les diaclases. Le marnage est de l'ordre de $9.1 \mathrm{~m}(\mathrm{SHOM})$, la zone du port est située à environ $+4 \mathrm{CM}$, la hauteur des vagues est généralement inférieure au mètre avec des périodes de houle inférieures à $10 \mathrm{~s}$, elles sont exceptionnellement supérieures à $5 \mathrm{~m}$ (la digue à talus toute voisine a été dimensionnée pour des houles de $3 \mathrm{~m}$ ).
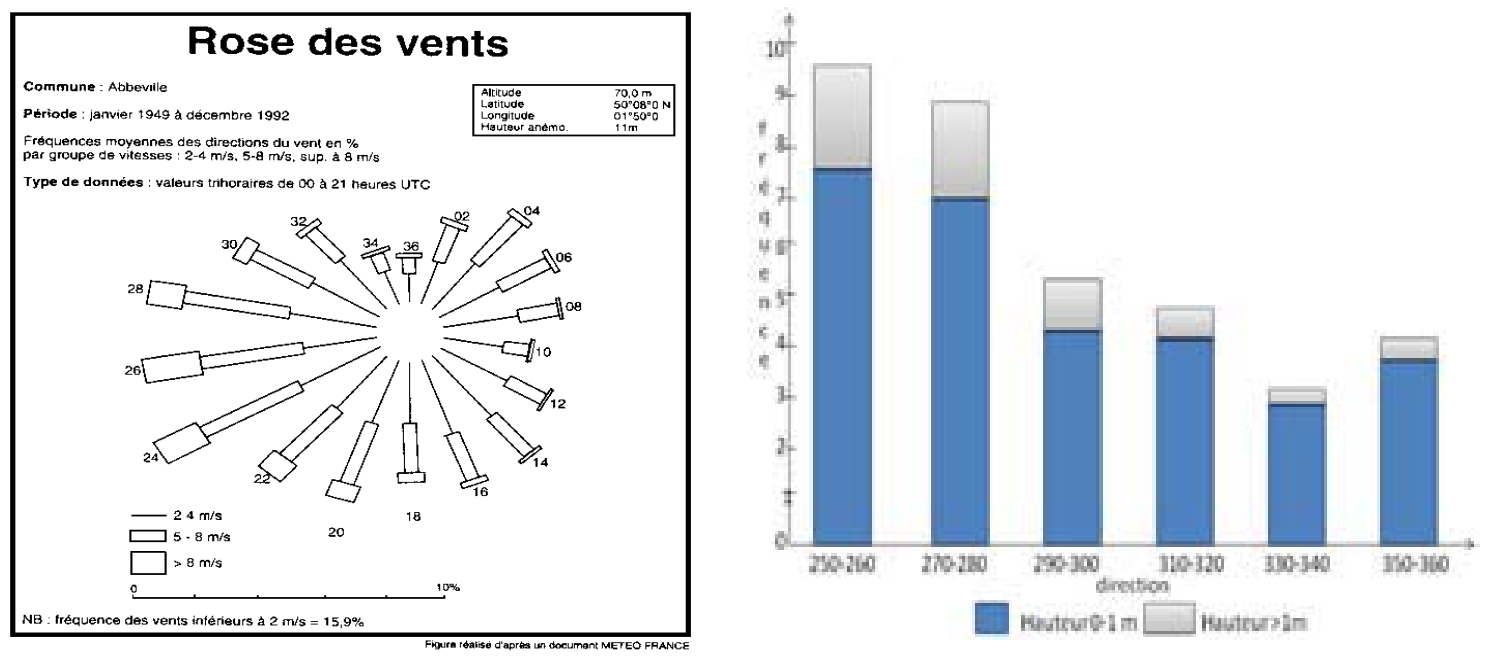

Figure 1. Rose des vents et fréquence des houles. La hauteur des vagues est inférieure à un mètre $80 \%$ du temps, elle dépasse rarement $3 \mathrm{~m}$. Les vents dominants sont d'Ouest, générant des houles et un transit dominant de même sens.

L'agitation correspond principalement à une mer de vent, la houle n'est pas complètement développée car les fetchs les plus importants n'excèdent pas $250 \mathrm{~km}$. Par vents de secteur ouest, les fronts d'onde sont obliques à la côte et la dérive littorale engendrée se dirige vers le Nord. Les vents de secteur nord-est sont moins fréquents et de force moindre; ils engendrent une dérive de sens opposée dirigée vers le Sud.

Le transit vers le Nord est prépondérant; il a lieu principalement pendant les mois d'hiver, quand les vents dominants sont de secteur ouest. Pour une houle d'amplitude $0,50 \mathrm{~m}$ et de période 5 secondes, arrivant à $20^{\circ}$ du trait de côte, des vitesses instantanées très variables, mais atteignant $4 \mathrm{~cm} / \mathrm{s}$ selon l'axe parallèle au rivage, ont été mesurées pour des galets de $7 \mathrm{~cm}$ (BEAUCHAMP, 1996).

Les galets ne sont accumulés que dans la partie supérieure de l'estran; l'épaisseur du cordon avoisine $10 \mathrm{~m}$ au niveau de l'amer de Cayeux, au Sud de la ville.

Les galets sont déplacés principalement à marée haute, quand le déferlement de type plongeant des vagues génère un jet de rive suffisamment puissant. A marée basse, la pente de la plage est très faible et le déferlement de type glissant s'effectue loin du 
rivage; le transport et la sédimentation n'affectent que les matériaux de faible taille, essentiellement les sables. La dérive littorale intervient également dans le transport des matériaux par traction sur le fond. Sa vitesse dépend notamment de l'amplitude et de la période de la houle. Par houle forte de 1,50 m d'amplitude, la vitesse de la dérive atteint $0,85 \mathrm{~m} / \mathrm{s}$ en surface.

L'étude du déplacement des galets le long du cordon a déjà été menée dans les années 50 par traçage radioactif (BEAUCHENE \& COURTOIS, 1957). Les galets ont été marqués au Titane 182, placés sur le cordon à $2 \mathrm{~km}$ au Nord d'Ault et suivis pendant 6 mois environ. La vitesse du déplacement a dépendu étroitement de l'agitation de la mer. La vitesse moyenne a varié de 2,3 m/jour (mer calme) à 14 m/jour (tempête).

Plus récemment, des mesures de déplacement au moyen de traceurs fluorescents ont été conduites au niveau de l'Amer de Cayeux, au Sud du bourg (BEAUCHAMP, 1996). La dispersion d'un stock de galets colorés a été suivie au cours d'un cycle de marée. Au delà, la dispersion, l'enfouissement des galets et l'usure de la peinture ne permettent plus d'obtenir des résultats représentatifs. Les résultats montrent que le déplacement se fait selon une direction parallèle au rivage avec des vitesses moyennes de déplacement variant de 1 à 3,6 m à l'heure pour un cycle de marée, avec une remontée de l'ensemble des galets vers le haut du cordon, la différence de niveau atteignant $7 \mathrm{~m}$. Le déplacement des galets se fait en général vers le Nord. Par vents de secteur Nord et Nord Est, l'obliquité des fronts de vagues et le sens de la dérive s'inversent; les galets sont ramenés vers le Sud. Le transit actuel de galet est relativement faible, de l'ordre de $2500 \mathrm{~m}^{3}$ /an (SOGREAH, 2009).
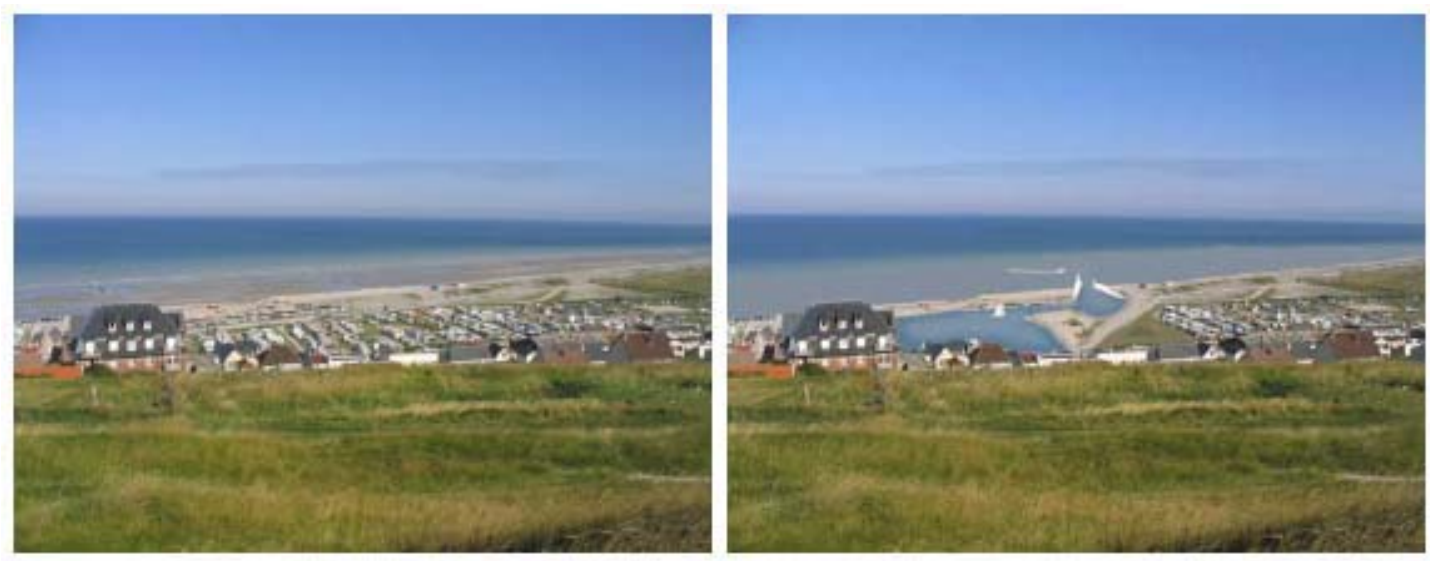

Figure 2. Montage photographique du projet de port vu depuis la colline. Le port se trouvera à l'emplacement actuel d'un camping, dans la commune de Woignarue. Cet emplacement dans la zone dite du «Hâble d'Ault » est sous le niveau des pleines mer, à la côte marine approximative de +4 CM. 


\section{XI ${ }^{\text {èmes }}$ Journées Nationales Génie Côtier - Génie Civil \\ Les Sables d'Olonne, 22-25 juin 2010}

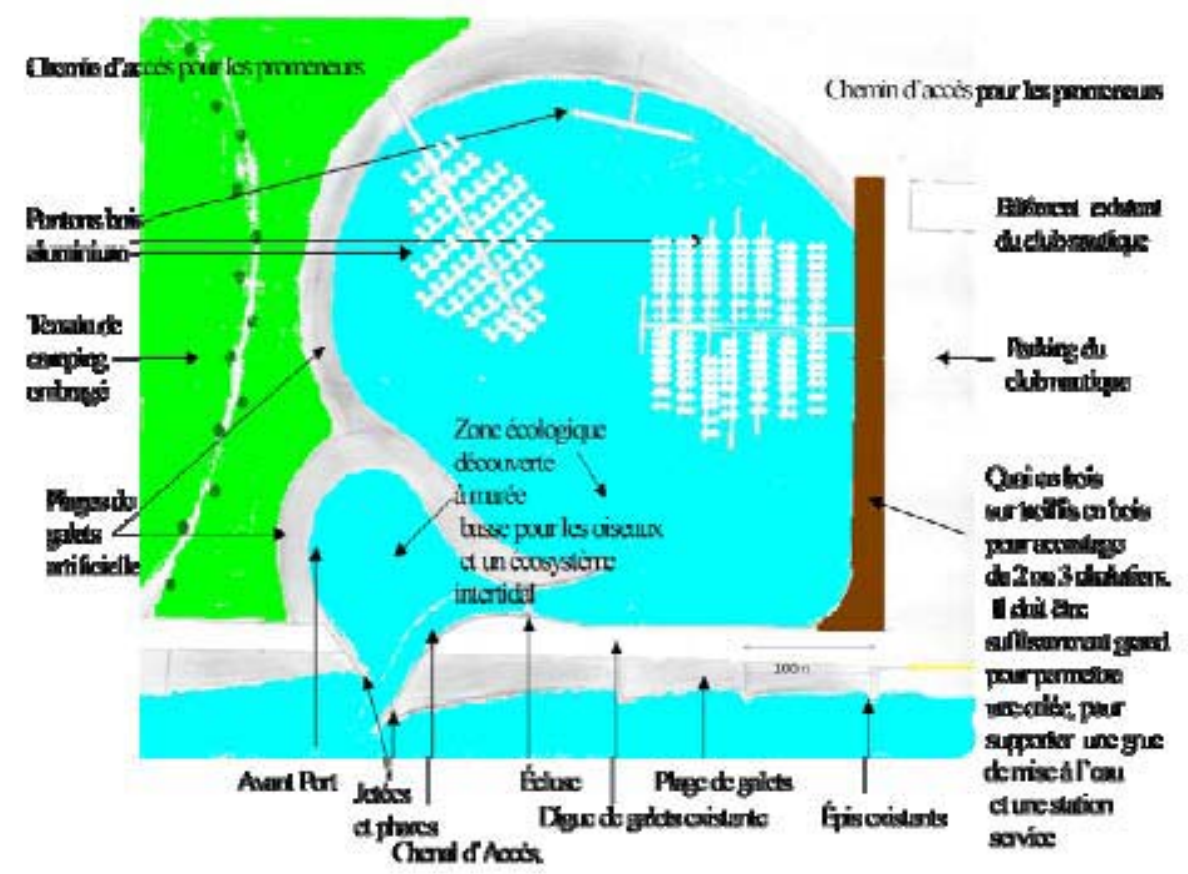

Figure 3. Esquisse du futur port.

\section{Calcul d'approche de houle}

Nous avons numérisé la bathymétrie de la carte 7416G du SHOM qui couvre la zone Sud de Boulogne-Sur-Mer jusqu'au Tréport. Cette numérisation compte environ 2000 points et couvre une zone de $900 \mathrm{~km}^{2}$. La bathymétrie aux abords de Ault est assez régulière et peu profonde, des profondeurs de $10 \mathrm{~m}$ sont atteintes à environ $5 \mathrm{~km}$ des côtes, de $20 \mathrm{~m}$ à une vingtaine de $\mathrm{km}$ des côtes, quelques bosses nommées "ridins" se caractérisent par une remontée des fonds de quelques mètres à une dizaine de mètre.

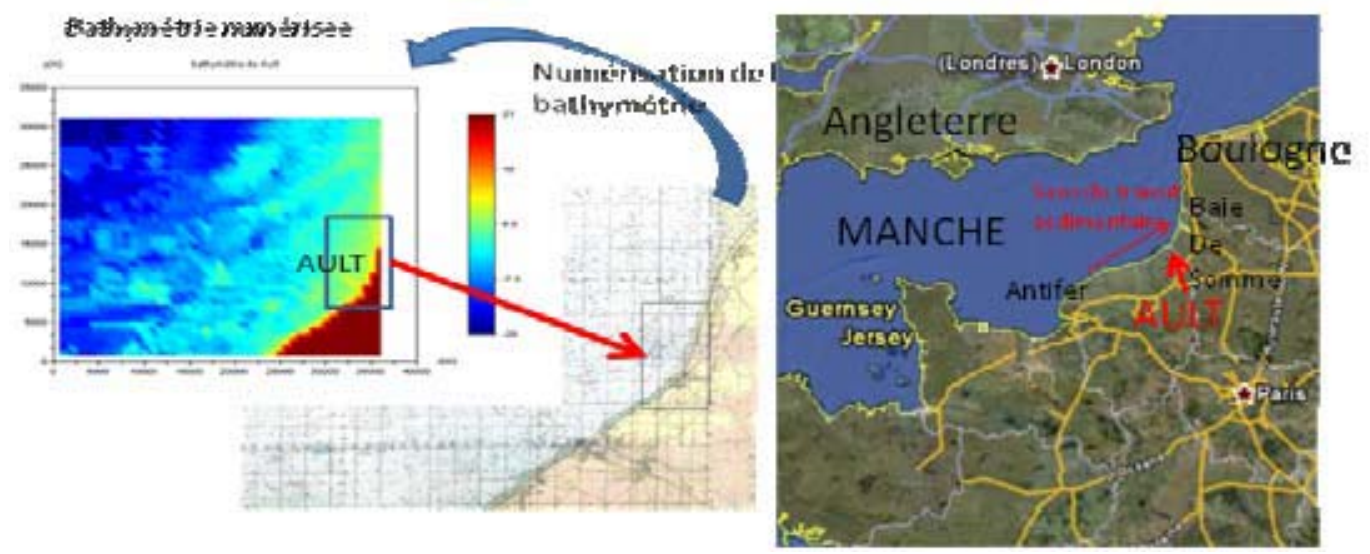

Figure 4. Gauche et Centre: Bathymétrie numérisée sur la carte SHOM 7416G pour faire les calculs d'approche. Droite : situation géographique d'Ault à la sortie de la Baie-de-Somme, dans le département de la Somme. 
Nous avons fait un calcul de propagation avec SWAN, une houle de $10 \mathrm{~s}$ de période et de $2 \mathrm{~m}$ venant de l'ouest (il y a rarement des houles de période si élevée).

Nous observons in-situ (observations empiriques sur site) une augmentation de l'amplitude au niveau de la côte entre 12000 m et 14000 m, c'est un peu plus au Sud que dans notre modélisation SWAN, une amplitude plus faible au sud de Ault, comme dans la modélisation.
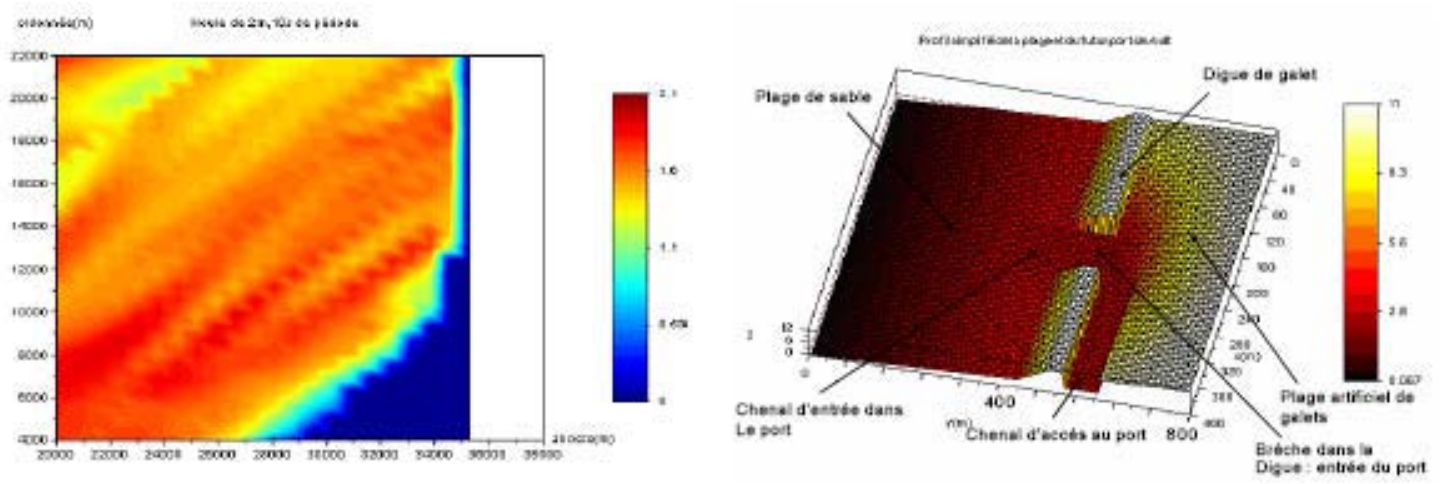

Figure 5. Gauche : Modélisation SWAN de la propagation d'une houle d'Ouest de $2 \mathrm{~m}$ de hauteur, 10 s de période sur une grande échelle. Droite : Modélisation de la bathymétrie de l'entrée du port.

\section{Etude de l'avant port avec SWAN}

L'objet de l'étude consiste à représenter une plage simplifiée de $800 \mathrm{~m}$ de long par 400 $\mathrm{m}$ de large. Le fond est supposé cylindrique, la profondeur varie de $12 \mathrm{~m}$ au large avec une pente de $1 \%$ sur $300 \mathrm{~m}$, puis de $10 \%$ sur $50 \mathrm{~m}$, cette dernière tranche représentant une plage de galet qui fait aussi office de digue de galet protégeant une zone dite de "bas champs" gagnée sur la mer.

Nous voulons savoir d'une part comment une houle de $1 \mathrm{~m}$ et de $4.6 \mathrm{~s}$ de période principale (Spectre de Jonswap) est affectée par le profil de la plage, et d'autre part comment cette houle se comporte si est creusée une brèche dans la digue de galet. La brèche ouvre sur une plage de galet artificielle que nous avons modélisé par une exponentielle, sur l'un des coté de l'exponentielle est creusé un chenal d'accès à un hypothétique port. Ces résultats montrent aussi que la bathymétrie dans l'avant port a des effets visibles sur la propagation des vagues. Du fait de la disposition du chenal, cet effet est positif car il dévie les vagues dans une direction opposée à l'entrée du port, il limite ainsi les effets nuisibles des vagues sur les infrastructures (écluse et ouvrages de défense) situées à proximité de l'entrée du port. Ils montrent aussi que la diffraction a un effet très prédominant sur tous les autres effets. L'entrée du port se comporte à peu de choses prés comme une fente étroite, les fronts de vague se répandent alors d'une manière assez homogène sur la largeur de l'avant port. 


\section{XI $I^{\text {èmes }}$ Journées Nationales Génie Côtier - Génie Civil}

Les Sables d'Olonne, 22-25 juin 2010

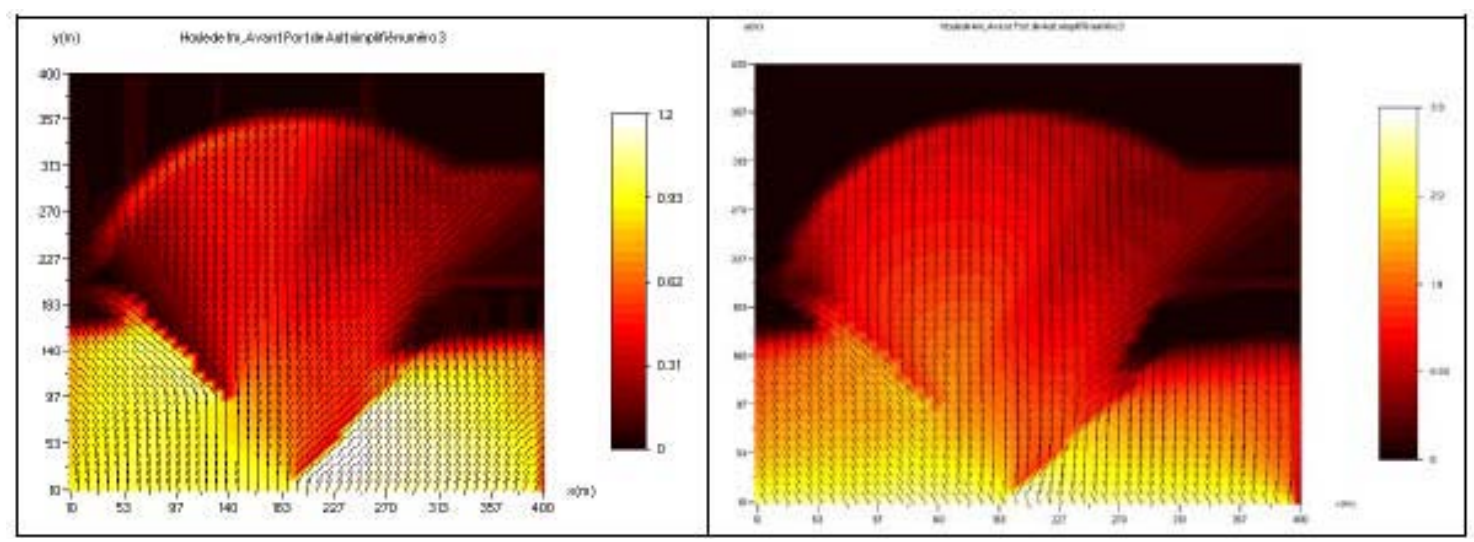

Figure 6. Modélisation des houles Nord-Nord ouest. (Remarque : les houles de Nord sont courtes et $4.6 \mathrm{~s}$ de période n'est pas un mauvais choix et Ouest, $8 \mathrm{~s}$ de période).

Cette modélisation confirme l'une de nos idées de base : il suffit de prolonger le chenal reliant l'avant port au port pour s'affranchir totalement des effets des vagues, et donc pour protéger et sauvegarder à moindre coût toutes les infrastructures des houles de tempêtes. Ces modélisations éclairent un autre problème associé aux vagues et aux courants : si la hauteur des vagues demeure relativement réduite dans l'avant port même par fortes houles, elles n'est pas négligeable et les flux d'énergie associés sont très importants dans la passe d'entrée alors que la profondeur est réduite, ce qui signifie que la densité d'énergie est grande. Par temps de tempête, ces flux d'énergie sont très importants sur tout le cordon littoral. Cette grande densité d'énergie se traduit par des courants très forts de l'ordre de quelques mètres par seconde dont l'intensité varie en fonction du niveau de la marée. Les courants de flot orientés du Sud vers le Nord sont généralement plus forts que ceux du Nord vers le Sud du reflux à cause de la dissymétrie de l'onde de marée. Ces effets de marée renforcent ou atténuent selon l'heure l'effet des courants dus aux vents et à l'obliquité de l'incidence de la houle, ces courants provoqués par les vents et les vagues sont parfois très puissants. La plage a tendance à concentrer par un effet proche de l'effet Venturi l'intensité du courant. Ces courants associés à l'effet de mise en suspension des sables et galets par les vagues de tempête sont aptes à modeler le profil de la plage et la bathymétrie de l'avant port en quelques heures de tempête. Une configuration optimale des dispositions relatives des jetées, des dimensions relatives des différentes parties de l'avant port et de la conformation de la bathymétrie sera cependant susceptible de maintenir dans un équilibre stable l'ensemble des éléments de l'avant port. Il sera nécessaire d'analyser l'agitation et la sédimentation dans le chenal :

- situation normale, les galets et le sable chutent dans le chenal et sont charriés sur de courtes distances sans transport de volume important vers l'intérieur du port ;

- situation de tempête annuelle : les galets peuvent être mis en suspension par l'action des vagues et pénétrer plus profond dans l'avant-port ; 
- situation de tempête décennale ou centennale: les plages Sud et Nord sont susceptibles d'être totalement remodelées (tempêtes de 1990 ayant entrainé l'inondation des Bas-Champs) avec une forte érosion, le flux d'énergie dans la passe est très important même si les vagues ne sont pas d'une taille extraordinaire, car elles sont très rapprochées les unes des autres. La plage de l'avant-port, si elle est mal équilibrée, est susceptible d'être remodelée. Une attention particulière devra être accordée à cette situation et l'avis de plusieurs experts devra être pesé, de même qu'une collecte de renseignements auprès des ports du Tréport et de Dieppe sera nécessaire.

Au-delà des considérations géométriques qui gouverneront l'établissement des rapports d'équilibre du port, l'utilisation appropriée de la puissance d'un bassin de chasse marée sera le moyen de prévenir tout comblement de l'avant port et de garantir le respect du transit littoral : il sera nécessaire de doser la force et de l'orienter de manière à obtenir l'effet désiré.

\section{Conclusion}

La construction d'un petit port de plaisance ne semble donc pas être impossible. Du fait de la grande réduction du transit littoral depuis quelques décennies à cause des différents ports entre Antifer et Ault, les galets ne devraient pas poser de problèmes. De plus, seules des jetées et une écluse seraient construits en dur, ce qui confèrerait au Port un caractère écologique appréciable.

\section{Références bibliographiques}

ANTEA (1996). Confortement de la digue des Bas Champs. Etude des possibilités de fourniture de galets et recherche de solutions alternatives. Rapport à D.D.E. Somme et SMACOPI, $24 \mathrm{p}$.

BEAUCHAMP J. (1996). Essai d'évaluation de la migration des galets le long de la côte picarde. Sociétés Humaines et milieux humides en Picardie, CTHS, Paris, pp 47-60.

BEAUCHENE P., COURTOIS G. (1957). Etude du mouvement des galets le long de la côte des Bas Champs de la Somme. Utilisation des traceurs radio-actifs. Cahiers océanographiques $\mathrm{n}^{\circ}$ 8, pp 613-625.

BINET C. (1994). La diminution de surface dans la Baie de Somme. Recherche des mécanismes. Trav. Départ. Géol. Univ. Picardie, 3, pp 101-110.

SOGREAH (2009). Confortement des zones urbanisées du Vimeu. Rapport. 